\title{
Loss function of SL (sekiguchi lesion) in the rice cultivar Minghui 86 leads to enhanced resistance to (hemi)biotrophic pathogens
}

Dagang Tian ${ }^{1 \dagger}$, Fang Yang ${ }^{2 \dagger}$, Yuqing $\mathrm{Niu}^{3 \dagger}$, Yan Lin ${ }^{1}$, Zaijie Chen ${ }^{1}$, Gang $\mathrm{Li}^{1}$, Qiong Luo ${ }^{4}$, Feng Wang ${ }^{1 *}$ and Mo Wang ${ }^{2,3,5^{*}}$

\begin{abstract}
Background: Serotonin, originally identified as a neurotransmitter in mammals, functions as an antioxidant to scavenge cellular ROS in plants. In rice, the conversion of tryptamine to serotonin is catalyzed by SL (sekiguchi lesion), a member of cytochrome P450 monooxygenase family. The $s /$ mutant, originated from rice cultivar Sekiguchi-asahi, exhibits spontaneous lesions, whereas its immune responses to pathogens have not been clearly characterized.

Results: Here we identified three allelic mutants of $S L$ in an indica rice restore line Minghui 86 (MH86), named as $5 /$ $\mathrm{MH}-1,-2$ and -3 , all of which present the typical lesions under normal growth condition. Compared with those in MH86, the serotonin content in sl-MH-1 is dramatically decreased, whereas the levels of tryptamine and L-trytophan are significantly increased. The sl-MH-1 mutant accumulates high $\mathrm{H}_{2} \mathrm{O}_{2}$ level at its lesion sites and is more sensitive to exogenous $\mathrm{H}_{2} \mathrm{O}_{2}$ treatment than the wild type. When treated with the reductant vitamin $\mathrm{C}(\mathrm{Vc})$, the lesion formation on $\mathrm{s} / \mathrm{MH}-1$ leaves could be efficiently suppressed. In addition, sl-MH-1 displayed more resistant to both the blast fungus and blight bacteria, Pyricularia oryzae (P. oryzae, teleomorph: Magnaporthe oryzae) and Xanthomonas oryzae pv. Oryzae (Xoo), respectively. The pathogen-associated molecular patterns (PAMPs)-triggered immunity (PTI) responses, like reactive oxygen species (ROS) burst and callose deposition, were enhanced in sl-MH-1. Moreover, loss function of SL resulted in higher resting levels of the defense hormones, salicylic acid and jasmonic acid. The RNA-seq analysis indicated that after $P$. oryzae infection, transcription of the genes involved in reduction-oxidation regulation was the most markedly changed in sl-MH-1, compared with MH86.
\end{abstract}

Conclusions: Our results indicate that $\mathrm{SL}$, involving in the final step of serotonin biosynthesis, negatively regulates rice resistance against (hemi)biotrophic pathogens via compromising the PTI responses and defense hormones accumulation.

Keywords: Rice, Serotonin, Reactive oxygen species, PAMP-triggered immunity, Defense hormones, Pyricularia oryzae

\footnotetext{
* Correspondence: wf@fjage.org; wangmo108@163.com

${ }^{\dagger}$ Dagang Tian, Fang Yang and Yuqing Niu contributed equally to this work.

'Biotechnology Research Institute, Fujian Key Laboratory of Genetic

Engineering for Agriculture, Fujian Academy of Agricultural Sciences, Fuzhou

350003, Fujian, China

${ }^{2}$ State Key Laboratory of Ecological Pest Control for Fujian and Taiwan Crops,

College of Life Science, Fujian Agriculture and Forestry University, Fuzhou

350002, Fujian, China

Full list of author information is available at the end of the article
}

\section{$\triangle B M C$}

(c) The Author(s). 2020 Open Access This article is licensed under a Creative Commons Attribution 4.0 International License, which permits use, sharing, adaptation, distribution and reproduction in any medium or format, as long as you give appropriate credit to the original author(s) and the source, provide a link to the Creative Commons licence, and indicate if changes were made. The images or other third party material in this article are included in the article's Creative Commons licence, unless indicated otherwise in a credit line to the material. If material is not included in the article's Creative Commons licence and your intended use is not permitted by statutory regulation or exceeds the permitted use, you will need to obtain permission directly from the copyright holder. To view a copy of this licence, visit http://creativecommons.org/licenses/by/4.0/. The Creative Commons Public Domain Dedication waiver (http://creativecommons.org/publicdomain/zero/1.0/) applies to the data made available in this article, unless otherwise stated in a credit line to the data. 


\section{Background}

In nature, plants are constantly exposed to a wide range of pathogenic microorganisms, thus they have developed a sophisticated innate immune system to protect themselves from infection [1]. PAMP-triggered immunity (PTI), activated upon recognition of conserved pathogenassociated molecular patterns (PAMPs) by plant cell membrane-localized pattern recognition receptors, can rapidly elicit plant defense responses, which includes calcium ions influx, reactive oxygen species (ROS) generation, callose deposition and stomatal closure et al [2]. Another branch of plant immunity is effector-triggered immunity (ETI), caused by direct or indirect perception of pathogen effectors via plant resistance (R) proteins, which is often associated with a hypersensitive response (HR) and renders plants the isolate-specific resistance [3]. As a typical feature of HR, programmed cell death (PCD), accompanied with production of ROS and phytoalexins, is employed to effectively halt the spread of pathogens within the initial penetration site [4]. On the other hand, activation of plant immune responses depends on dramatic changes in the cellular reduction-oxidation (redox) status, resulting in the reprogramming of the transcriptome and the establishment of both local and systemic defense [5]. Upon being infected, the raised oxidative stress in plant cells is essential for HR formation and development [6].

Serotonin (5-hydroxytryptamine) is originally known as a neurotransmitter controlling fundamental physiological processes, such as mood, sleep and anxiety, in mammals [7]. Since its first identification in the Mucuna pruriens, serotonin has been found widely distributed in the plant kingdom and involved in regulation of diverse physiological processes $[8,9]$. Serotonin biosynthesis in plants occurs as that tryptophan is converted into tryptamine by tryptophan decarboxylase, following with the catalysis of tryptamine to serotonin by tryptamine 5hydroxylase [10]. Serotonin is readily oxidized and functions as an antioxidant to scavenge cellular ROS [11]. And the antioxidant activity of serotonin is reported to far exceed that of tryptophan, tryptamine and its derivatives [9]. Thus, it is conceivable that serotonin plays a role in plant innate immunity via regulating cellular redox status. Moreover, serotonin could also be incorporated into the plant cell wall for strengthening the mechanical barrier against pathogens [12].

Rice (Oryza sativa) is the staple food for more than half of the world's population. Rice blast and bacterial blight, caused by the hemibiotrophic fungal pathogen Pyricularia oryzae (P. oryzae) and the biotrophic bacterial pathogen Xanthomonas oryzae pv. Oryzae (Xoo), respectively, are the most devastating rice diseases [13]. Increasing reports indicate that the serotonin pathway in rice is involved in disease resistance, and most of studies are based on characterizing a lesion mimic mutant, sekiguchi lesion (sl), originated from the rice cultivar Sekiguchi-asahi. The $S L$ gene, cloned by Fujiwara et. al in 2010, encodes CYP71P1, belonging to the cytochrome P450 monooxygenase family [14]. SL possesses tryptamine 5-hydroxylase enzyme activity and can catalyze the conversion of tryptamine to serotonin in rice. As this reason, the accumulation of serotonin after Bipolaris oryzae (B. oryzae, a necrotrophic pathogen) infection was abolished in $s l$ mutant, and $s l$ displayed increased susceptibility to $B$. oryzae [12]. However, the responses of $s l$ mutant to biotrophic pathogens infection and the mechanisms of SL involved in plant immunity are still ambiguous.

In this study, we identified the $s l$ mutants in MH86 background $(s l-M H)$ and found that the lesions of $s l$ $M H-1$ is caused by excessive accumulation of ROS, which could be suppressed by the reductant treatment. The absence of SL results in the enhanced PTI responses and high resting levels of defense hormones, and thus the broad-spectrum resistance against $P$. oryzae and Xoo.

\section{Results \\ Identification of the $s /$ mutants in MH86 background}

We have identified a rice runaway-cell death mutant, $r c d 1-1$, in an indica rice restore line Minghui 86 (MH86). This mutant spontaneously exhibits orangecolored lesions on its leaves when grown in field or greenhouse (Fig. S1). Through map-based cloning, a G to $\mathrm{T}$ mutation was found at 1205 nucleotide of SL ORF, which leads to the $370 \mathrm{Arg}$ mutated to Leu [15]. Two allelic mutants, $r c d 1-2$ and $r c d 1-3$, were obtained by ${ }^{60} \mathrm{Co} \sim \gamma$-ray radiation, which carries $\mathrm{C} 85$ and A1420 deletion in $S L$ coding region, respectively (Fig. S2). Both $r c d 1-2$ and $r c d 1-3$ spontaneously present sekiguchi lesions, similar with $r c d 1-1$ (Fig. S1). Therefore, hereafter we named $r c d 1-1, r c d 1-2$ and $r c d 1-3$ mutants as $s l$ $M H-1, s l-M H-2$ and $s l-M H-3$, respectively.

To investigate difference of the metabolites levels in serotonin biosynthesis pathway in $s l-M H-1$ mutant and MH86, the contents of serotonin, tryptamine and Ltrytophan from the leaves of 8-week-old plants grown in a greenhouse with natural light were measured. The data indicated that serotonin level was significantly decreased in $s l-M H-1$ compared with MH86, whereas tryptamine and L-trytophan, the upstream metabolites in serotonin biosynthesis, were accumulated to significantly higher levels in $s l-M H-1$ (Fig. 1), proving the function of SL in catalyzing the conversion of tryptamine to serotonin. Moreover, we found that the content of L-glutamine in sl-MH-1 was significantly higher than that in MH86 (Fig. S3). Taken together, our results indicate that mutation of $S L$ in MH86 background also results in the 

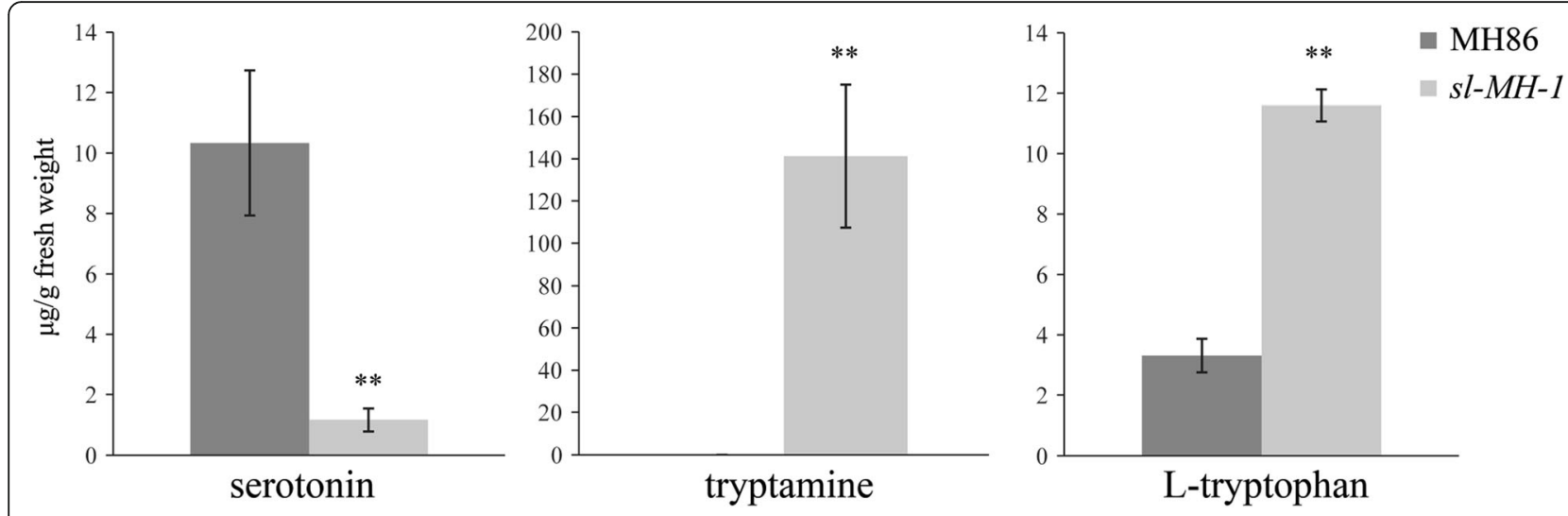

Fig. 1 Levels of serotonin, tryptamine and L-tryptophan in MH86 and sl-MH-1. The contents of serotonin, tryptamine and L-tryptophan in the leaves of 8-week-old MH86 and sl-MH-1 plants were measured by Ultra High Performance Liquid Chromatography (UHPLC). Bars represent mean values \pm standard error (SE) from five biological replicates. Statistically significant difference was indicated by ** $(p<0.01$, Student's $t$-test)

typical sekiguchi lesion and compromised serotonin biosynthesis.

\section{Endogenous oxidative stress results in lesion formation on $\mathbf{s l}-\mathrm{MH}-1$ leaves}

To determine the relationship between cell death and ROS accumulation in absence of SL, we stained the leaves of 8-week-old sl-MH-1 and MH86 with trypan blue and 3,3-diaminobenzidine tetrahydrochloride (DAB) to detect cell death and $\mathrm{H}_{2} \mathrm{O}_{2}$ accumulation, respectively. As shown in Fig. 2a, compared with MH86, the lesion sites on $s l-M H-1$ leaves (shown by the dark trypan blue staining) displayed more $\mathrm{H}_{2} \mathrm{O}_{2}$ accumulation as indicated by DAB staining (dark brown color). To assess the defects of $s l-M H-1$ responding to exogenous ROS stress, $s l-M H-1$ and MH86 seeds were germinated on Murashige and Skoog (MS) medium with or without $\mathrm{H}_{2} \mathrm{O}_{2}$. After kept in a $28^{\circ} \mathrm{C}$ growth chamber with 12 -h light for 1 week, we found that there was no significant difference between $s l-M H-1$ and MH86 seedlings on MS medium without $\mathrm{H}_{2} \mathrm{O}_{2}$ (Fig. 2b). The $\mathrm{H}_{2} \mathrm{O}_{2}$ application could retard the growth of both sl-MH-1 and MH86 seedlings, whereas $s l-M H-1$ seedlings displayed more stressed (with leaves turning yellow) than the wild type to $\mathrm{H}_{2} \mathrm{O}_{2}$ treatment (Fig. 2b). Therefore, $S L$ mutation leads to high endogenous ROS accumulation and increased sensitivity to exogenous ROS stress.

In order to further determine whether lesion of $\mathrm{sl}$ $M H-1$ is resulted from its high internal ROS accumulation, we treated the mutant with the antioxidant Vitamin $\mathrm{C}$ (VC, ascorbic acid), which is one of the major redox buffers in plant cell and functions as an antioxidant to conduct ROS detoxification [16]. And it has been reported that exogenous application of VC could alleviate oxidative stress in rice induced by abiotic stress [17]. 3week-old sl-MH-1 seedlings grown in green house, whose leaves did not exhibit visible lesions, were treated with $0.1 \% \mathrm{VC}$ or water (as control) by spraying for 10 days. Then the lesion numbers on the top second and third leaves were counted. As shown in Fig. 2c and Table 1, the lesion numbers on $s l-M H-1$ leaves was significantly decreased by $\mathrm{VC}$ treatment, indicating that exogenous antioxidant application could suppress $s l-M H-1$ lesion formation. Taken together, our data suggest that the cell death in absence of SL is caused by high endogenous ROS accumulation.

\section{sl-MH-1 mutant displays enhanced resistance to $P$. oryzae and $\mathrm{XoO}$}

As ROS production is an important defense response of plants to pathogen invasion, we wondered whether $s l$ MH-1 mutant was more resistant to rice pathogens. Firstly, we challenged 3-week-old sl-MH-1 and MH86 seedlings with conidial spores of the compatible blast fungal isolate, FJ-1, by spraying. As shown in Fig. 3a, inoculation with FJ-1 caused typical disease symptom on MH86 leaf, whereas $s l-M H-1$ mutant exhibited sekiguchi lesions at the FJ-1 infection sites. To quantify the resistance of $s l-M H-1$ to the blast fungus, we carried out punch inoculation on sl-MH-1 and MH86 leaves and investigated the relative fungal biomass within the infected region, which showed that the sl-MH-1 mutant supported significantly less blast fungus growth than MH86 (Fig. 3b). In order to further monitor the process of $M$. oryzae invasion in $s l-M H-1$ and MH86, a rice leaf sheath inoculation assay was performed. It was found that the invasive hyphae (IH) in MH86 could extend to the neighboring cells of the first infected cell at $48 \mathrm{~h}$ post inoculation (hpi) and further extended to the adjacent cells at 72 hpi (Fig. 3c). However, at both 48 and 72 hpi, the $\mathrm{IH}$ was still constricted in the first infected cell of $s l$ $M H-1$ (Fig. 3c). Although the IH could elongate from 


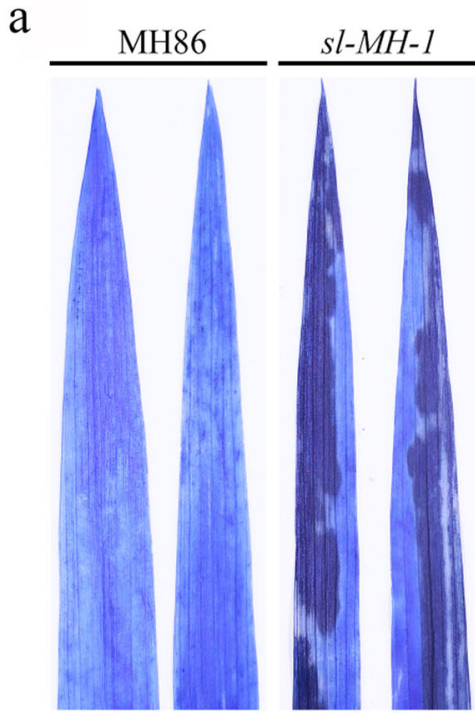

trypan blue staining

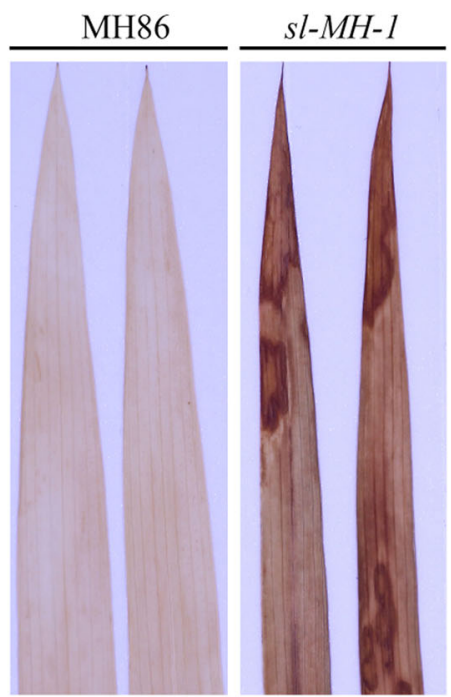

DAB staining

b

MH86

sl-MH-1

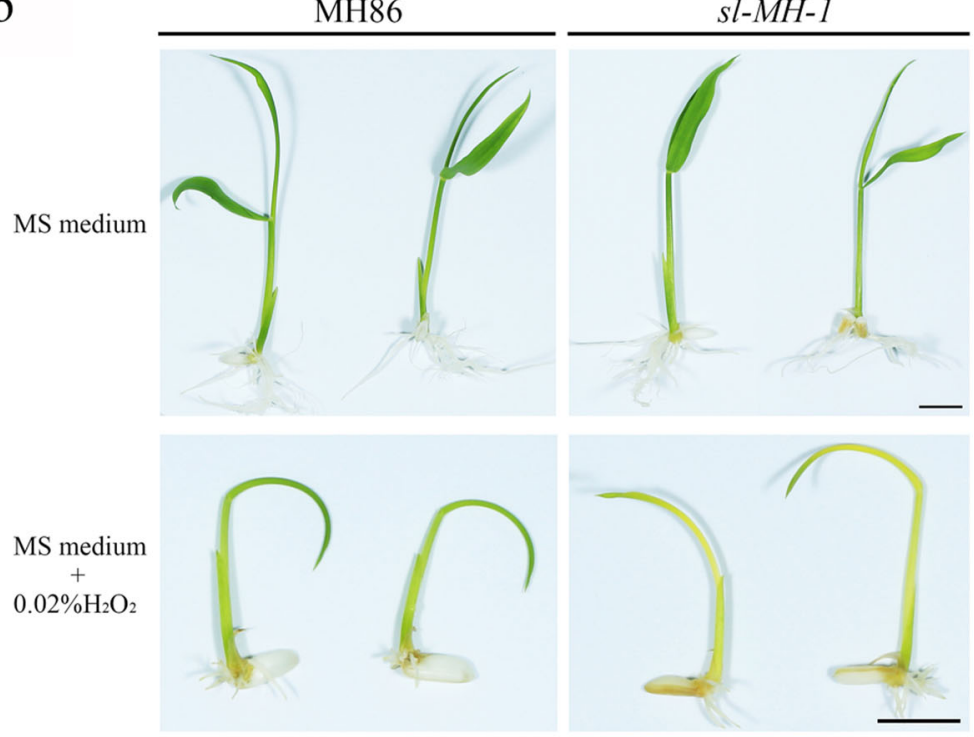

C

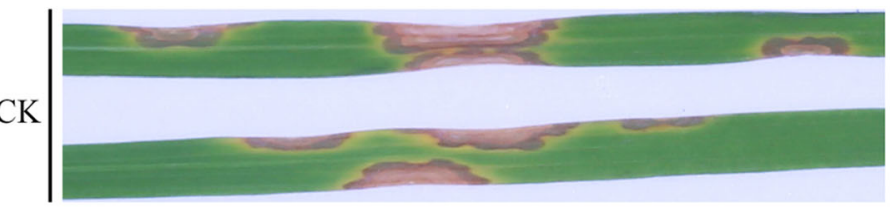

$0.1 \% \mathrm{VC}$

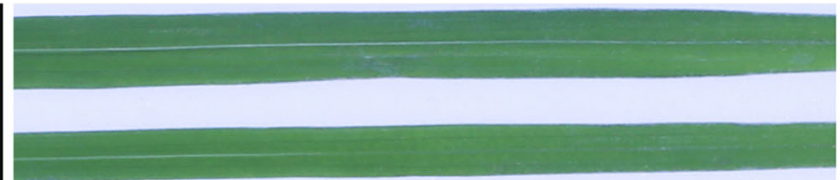

Fig. 2 Responses of sl-MH-1 to exogenous $\mathrm{H}_{2} \mathrm{O}_{2}$ and VC treatments. a Trypan blue (left) and DAB (right) staining of the 8-week-old sl-MH-1 and MH86 leaves to detect cell death and $\mathrm{H}_{2} \mathrm{O}_{2}$ accumulation, respectively. $\mathbf{b}$ sl-MH-1 and MH86 seeds were germinated on MS medium with or without $0.02 \% \mathrm{H}_{2} \mathrm{O}_{2}$. The seedlings were imaged after kept in a growth chamber for 7 days. Bars $=1 \mathrm{~cm}$. c. The leaves of $s /-M H-1$ plants were imaged after treated with $0.1 \%$ VC for 10 days. CK means water treatment as control 
Table 1 Lesion numbers on the sl-MH-1 leaves after VC and CK treatments

\begin{tabular}{lll}
\hline treatment & \multicolumn{2}{l}{ average number of lesions per leaf } \\
\cline { 2 - 3 } & top second leaf & top third leaf \\
\hline CK (86 plants) & $1.33 \pm 1.38$ & $1.24 \pm 1.41$ \\
$0.1 \% \mathrm{VC}$ (42 plants) & $0.05 \pm 0.22^{* *}$ & $0.70 \pm 0.26^{* *}$ \\
\hline
\end{tabular}

** means statistically significant with $p<0.01$ with One-way analysis of variance (ANOVA)
48 to $72 \mathrm{hpi}$ in $s l-M H-1$ cells, the infection rate of blast fungus was markedly slower than that in MH86. Taken together, these results indicate that knockout of $S L$ enhances rice resistance to blast fungus.

To determine whether $s l-M H-1$ mutant possesses the increased resistance to bacterial pathogens, we further inoculated sl-MH-1 and MH86 plants with Xoo. As shown in Fig. 4, via comparing blight lesion length and bacterial population at 14 days after inoculation, it was found that $s l-M H-1$ indeed displayed enhanced resistance to blight bacteria as well. Furthermore, to support the resistant phenotypes of $s l-M H-1$ is resulted from loss function of SL, another allelic mutant, $s l-M H-3$, was subjected to the above inoculation assays. The results

\section{a}

MH86

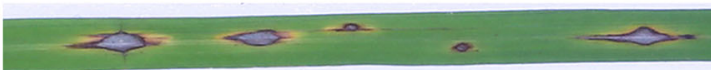

$s l-M H-1$

b

MH86
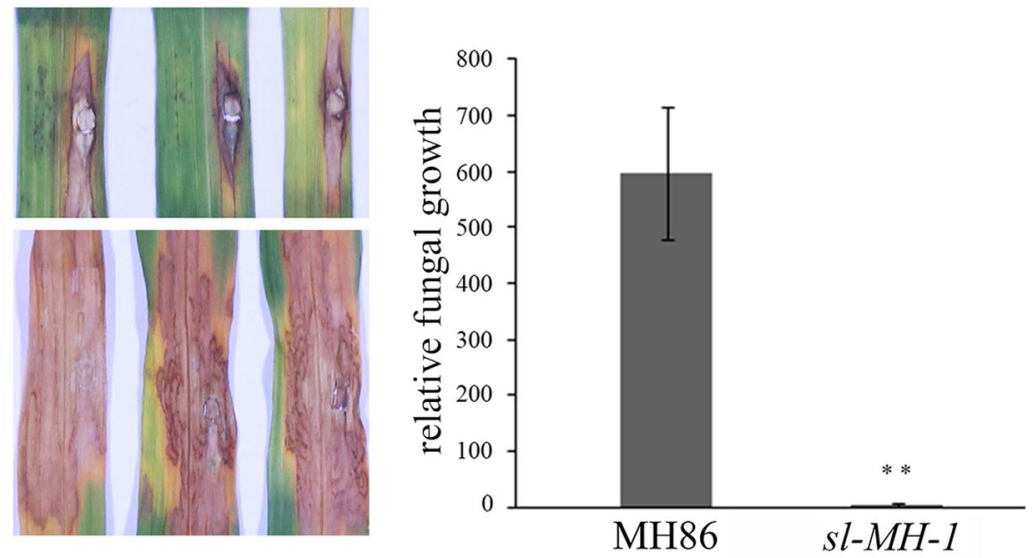

C

48 hpi
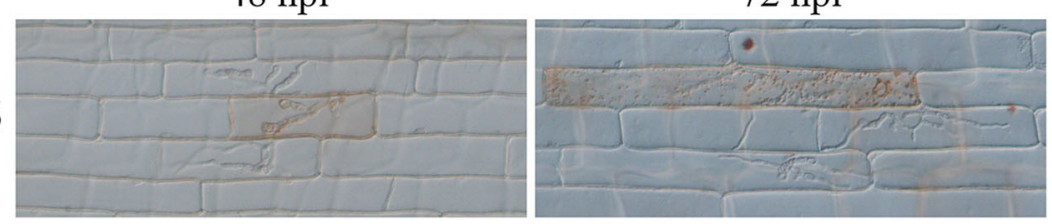

$s l-M H-1$
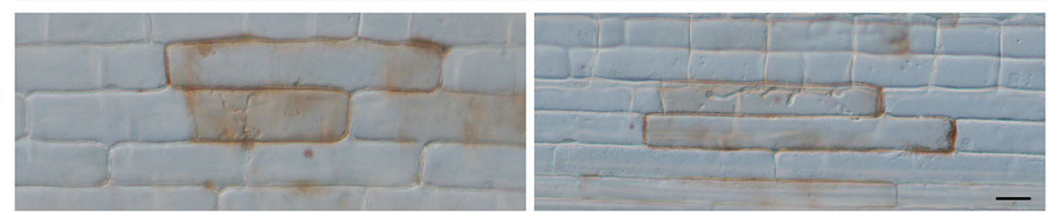

Fig. 3 sl-MH-1 mutant displays increased resistance to P. oryzae. a 3-week-old MH86 and sl-MH-1 seedlings were inoculated with P. oryzae conidia by spraying. At 7 days post inoculation (dpi), the diseased leaves were imaged. b Punch inoculation with P. oryzae conidia was carried out on the leaves of 4-week-old MH86 and sl-MH-1 plants. The infected leaves were photographed at $9 \mathrm{dpi}$ (left); meanwhile fungal biomass was measured to quantify the relative $P$. oryzae growth in $\mathrm{MH} 86$ and sl-MH-1 leaves (right). Bars represent mean values \pm SD from three biological replicates. Statistically significant difference was indicated by ** $(p<0.01$, Student's $t$-test). c. Leaf sheathes of MH86 and sl-MH-1 were inoculated with $P$. oryzae conidia to monitor the infection process. The infected cells were imaged at 48 and 72 hpi with a microscope under bright field. Bar $=20 \mu \mathrm{m}$ 
a

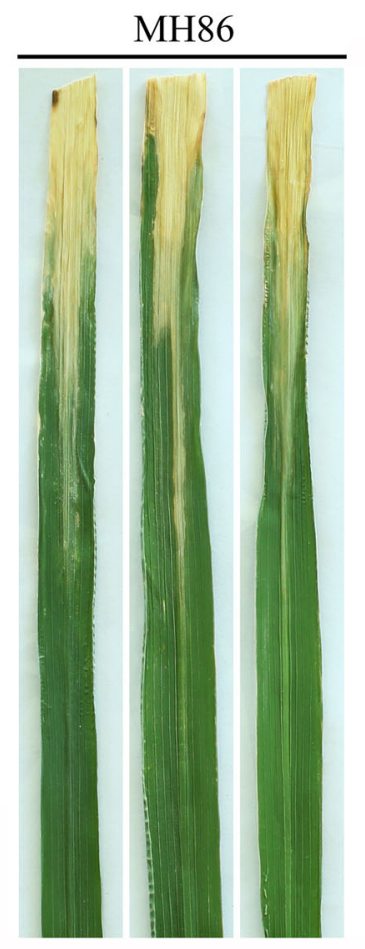

$\mathrm{b}$

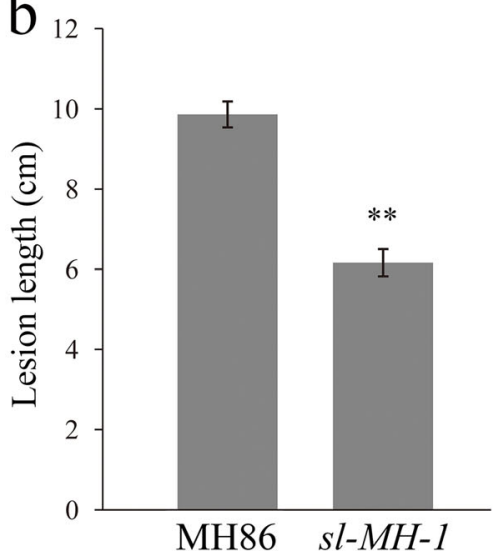

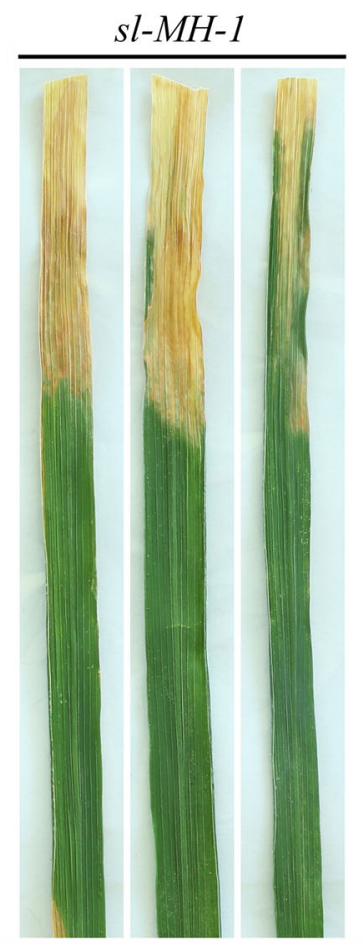

C

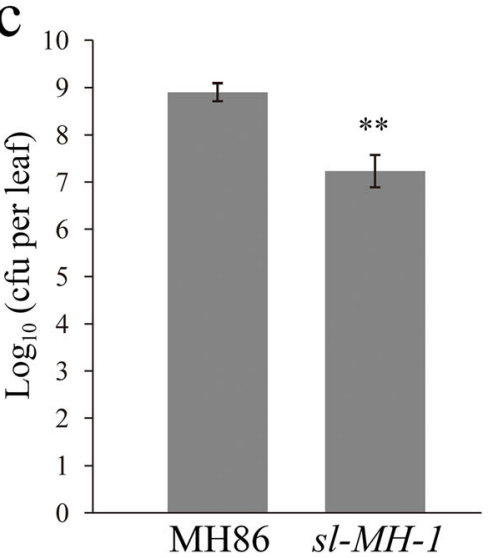

Fig. $4 \mathrm{sl}-\mathrm{MH}-1$ mutant showed enhanced resistance to $X_{0 O}$. a $\mathrm{MH} 86$ and $\mathrm{sl}-\mathrm{MH}-1$ plants were inoculated with $\mathrm{XoO}_{\mathrm{O}}$ at the tilling stage. The infected leaves from three independent $\mathrm{MH} 86$ or sl-MH-1 plants were imaged at $14 \mathrm{dpi}$. b Blight lesion length on sl-MH-1 and $\mathrm{MH} 86$ leaves were measured. Bars represent mean values \pm SD ( $n=6$, from three independent plants). c Blight bacterial populations were counted 14 dpi with bars representing mean values \pm SD from three independent $\mathrm{MH} 86$ or sl-MH-1 plants. Cfu means colony-forming units. Statistically significant difference in $b$ and $c$ was indicated by ** $(p<0.01$, Student's t-test)

showed that similar with $s l-M H-1, s l-M H-3$ displayed more resistance to $P$. oryzae and Xoo (Fig. S4a-d). Therefore, SL negatively regulates rice broad-spectrum resistance to the (hemi)biotrophic pathogens.

\section{$S L$ mutation leads to enhanced PTI responses}

In order to decipher the mechanism of enhanced resistance by SL deletion, the PTI responses of $s l-M H-1$ and MH86 were analyzed. We firstly measured the levels of ROS burst in $s l-M H-1$ and MH86 upon flg22 and chitin treatments. It was found that ROS burst could be stimulated in both $s l-M H-1$ and MH86 leaves by the PAMPs treatments, whereas the levels of ROS production were significantly higher in $s l-M H-1$ than MH86 at the indicated time points (Fig. 5a). Additionally, the callose deposition on $s l-M H-1$ and MH86 leaves after chitin treatment was investigated. As shown in Fig. 5b, compared with MH86, there was significantly more callose deposited on $s l-M H-1$ leaves. And the enhanced callose deposition was similarly observed on $s l-M H-3$ leaves (Fig. S4e). Furthermore, transcriptional profile of the PTI-related defense gene $K S 4$ was investigated in $s l-M H$ - 

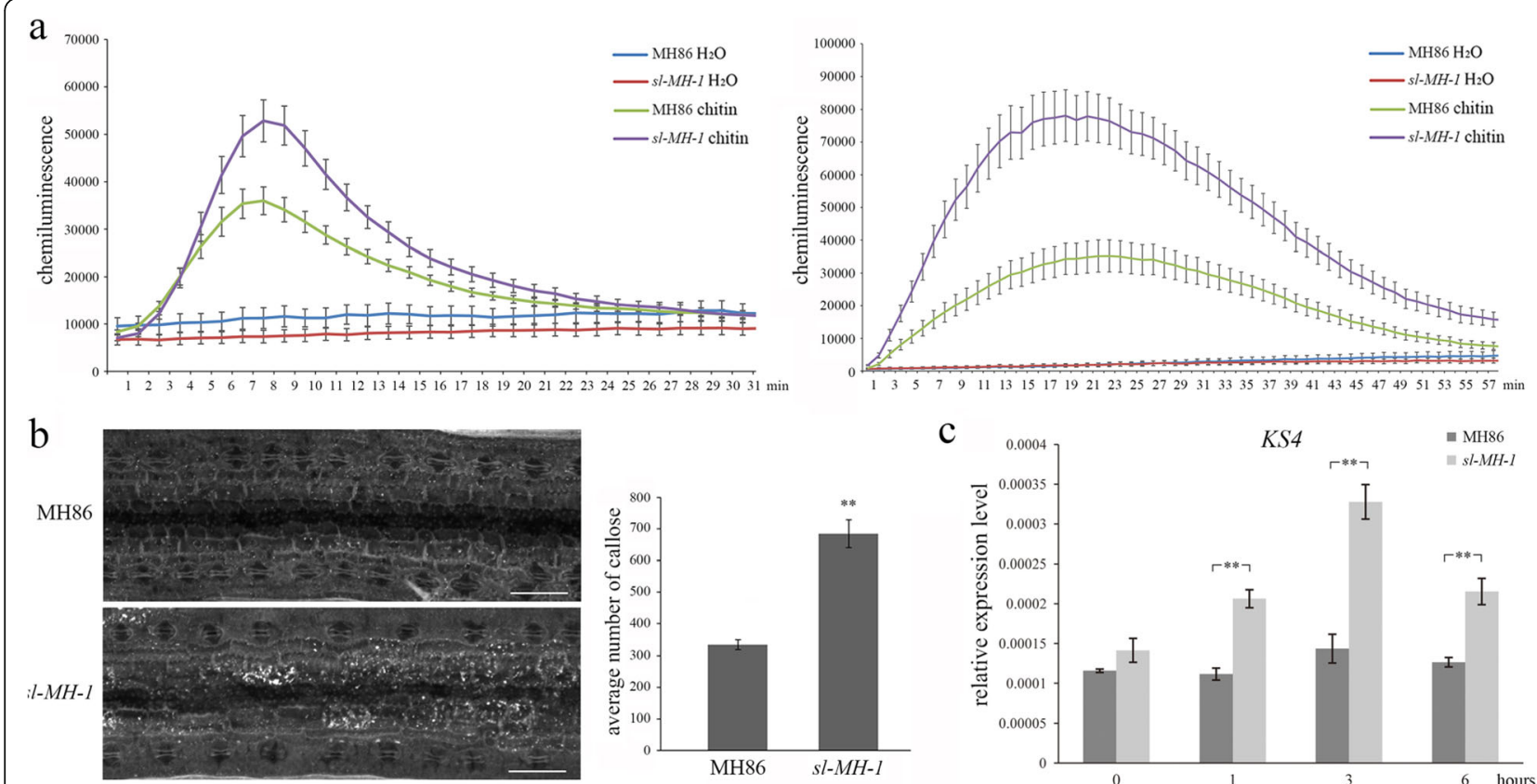

Fig. 5 PTI responses are enhanced in sl-MH-1 mutant. a ROS burst from MH86 and sl-MH-1 leaf discs treated with $400 \mathrm{nM}$ chitin or $500 \mathrm{nM}$ flg22 was detected at the indicated time points. Error bars represent the SE $(n=8)$. The data are from one of three independent experiments performed with similar results. B Callose deposition on MH86 and sl-MH-1 leaves after chitin treatment was imaged with a microscope under UV light (left). Number of the callose deposition per view was analysed by Image J (right). Bars represent mean values \pm SE $(n=5)$. Statistically significant difference was indicated by ${ }^{* *}(p<0.01$, Student's $t$-test). This assay was performed in three independent replicates with similar results. c The relative transcriptional levels of KS4 in 3-week-old MH86 and sl-MH-1 leaves at the indicated time points after treated with $400 \mathrm{nM}$ chitin. Rice ubiquitin-encoding gene (UBQ) was used as the internal control. Bars represent mean values $\pm S D(n=3)$. Statistically significant difference was indicated by ** $(p<0.01$, Student's $t$-test). This assay was performed in two independent replicates with similar results

1 and MH86 before and after chitin treatment. KS4 is a diterpene cyclase enzyme involving in momilactone biosynthesis $[18,19]$. We found that at all the indicated time points after chitin treatment, the transcriptional levels of KS4 in $s l-M H-1$ were significantly higher than those in MH86 (Fig. 5c). Taken together, our data demonstrate that loss function of SL enhances rice PTI responses.

\section{sl-MH mutants accumulate higher resting levels of defense hormones}

Salicylic acid (SA) and jasmonic acid (JA) are the major phytohormones involved in defense against pathogens. To assess whether $S L$ mutation alters contents of these defense hormones, we detected the levels of free SA, JA and JA-isoleucine (JA-Ile, the active form of JA) in 6 weeks-old $s l-M H-1$ and MH86 plants, when the lesions were fully appeared on $s l-M H-1$ leaves. As shown in Fig. 6, the free SA level in $s l-M H-1$ was about twice as high as that in MH86; both JA and JA-Ile contents in $s l$ $M H-1$ were burst to approximately 30 times higher than those in MH86. Meanwhile, levels of SA, JA and JA-Ile were also measured in $s l-M H-3$ to confirm that the high accumulation of the defense hormones was resulted from $S L$ mutation. It was found that $s l-M H-3$ accumulated similar level of free SA with $s l-M H-1$ and even higher levels of JA and JA-Ile than those in $s l-M H$ 1 (Fig. 6). Moreover, the levels of defense hormones were also measured in 3 weeks-old sl-MH-1 and MH86 plants, when the lesions were not observed on $s l-M H-1$ leaves. As shown in Fig. S5, the free SA level in 3-weekold $s l-M H-1$ seedlings was similarly about two-fold higher than that in MH86; JA-Ile content in sl-MH-1 was about 3 times higher than that in MH86, whereas no significant difference of JA level was detected between 3-week-old $s l-M H-1$ and MH86. These results suggest that $S L$ mutation leads to constantly more SA production and dramatically increased JA and JA-Ile contents along with the lesion appearance.

\section{$S L$ mutation mainly alters transcription of the genes in} reduction-oxidation pathway upon blast fungus infection To determine the difference of gene transcription in $s l$ mutant and wild type in response to pathogen infection, we carried out transcritome analysis in sl-MH-1 and MH86 plants with and without $P$. oryzae inoculation. The leaf tissues of $s l-M H-1$ and MH86 were harvested at 48 hpi with FJ-1 to conduct RNA-seq analysis, water treatment was used as control (mock). The number of significant differentially expressed loci (SDEL) in $s l-M H-1$-mock 


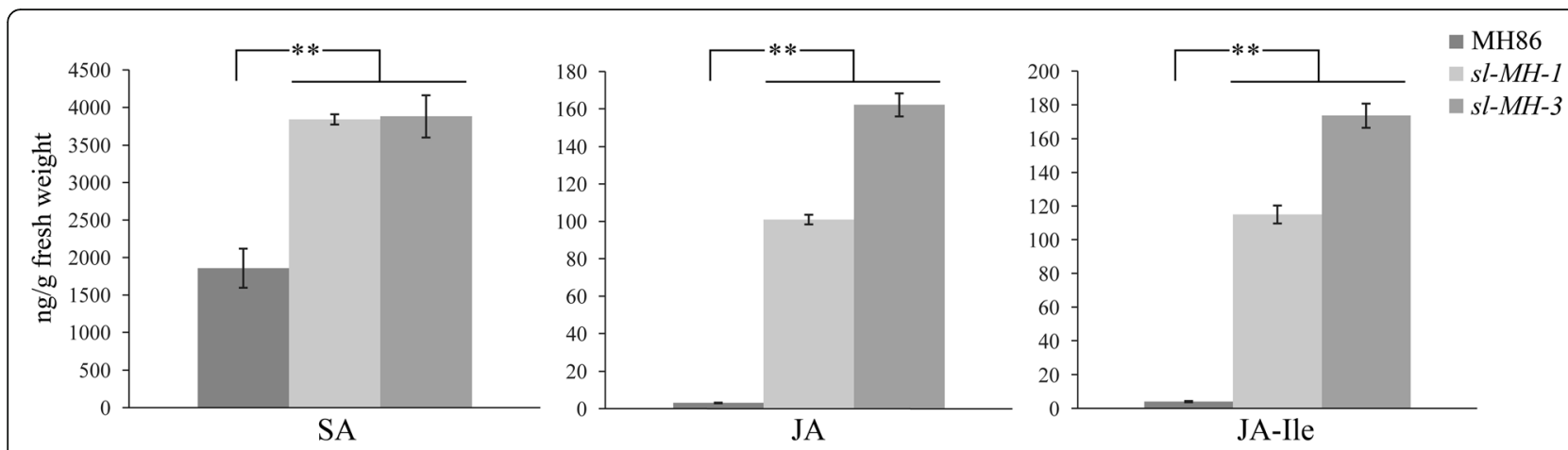

Fig. 6 Contents of SA, JA and JA-lle in MH86 and sl-MH mutants. The resting levels of SA, JA and JA-lle in 6-week-old MH86, sl-MH-1 and sl-MH-3 plants were measured by UPLC. Bars represent mean values \pm SD from three biological replicates. Statistically significant difference was indicated by ${ }^{* *}(p<0.01$, Student's $t$-test $)$

compared to MH86-mock (sl-MH-1-mock/MH86-mock) and sl-MH-1-48hpi compared to MH86-48hpi $(s l-M H-1$ 48hpi/MH86-48hpi) with fold change $\geq 2$ or $\leq 0.5$ were 95 and 149, respectively (supplementary Table S1 and Table S2). We used Gene Ontology enrichment analysis to investigate the transcript profiles in various biological processes of sl-MH-1 and MH86 following blast fungus infection. The results indicated that compared with
MH86, the most markedly affected biological process in $s l-M H-1$ is the redox pathway (Fig. 7a). The significant differentially expressed genes (DEGs) related to redox regualtion were 10 and 23 in sl-MH-1-mock/MH86-mock and sl-MH-1-48hpi/MH86-48hpi, respectively, whose transcript levels were displayed as heatmaps shown in Fig. $7 \mathrm{~b}$. There are five redox-related genes had similar trend of transcriptional change in both sl-MH-1-mock/ a

$\mathrm{GO}$ enrichment in biological process:
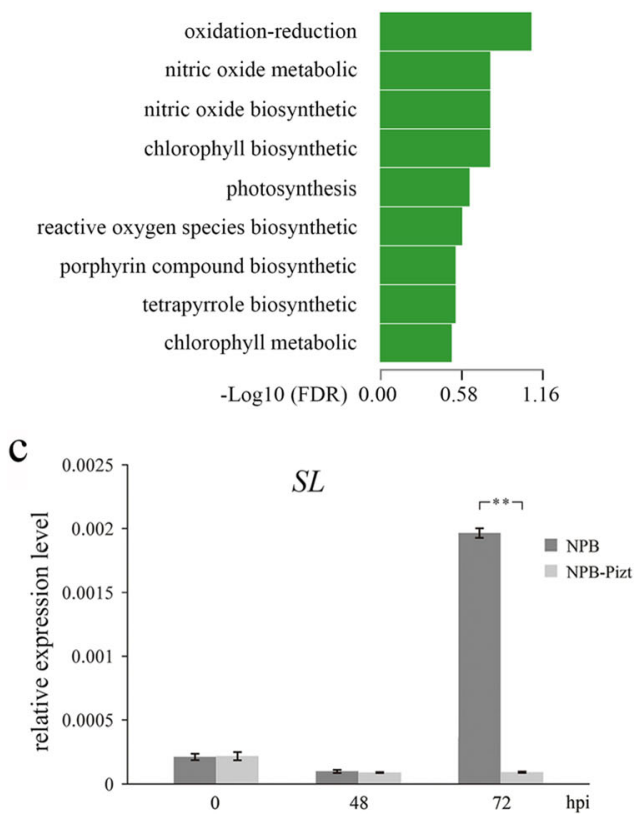

\section{b}

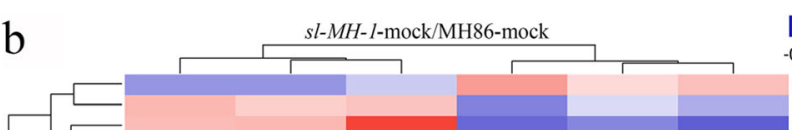
$0.4 \quad 0.4$ Os01g0357100 Os01g0591000 Os01g0963000 : Os05g0465900 Os06g0208151 • Os06g0640800 Os07g0520300 •

H86-3 sl-MH-l-48hpi/MH86-48hpi

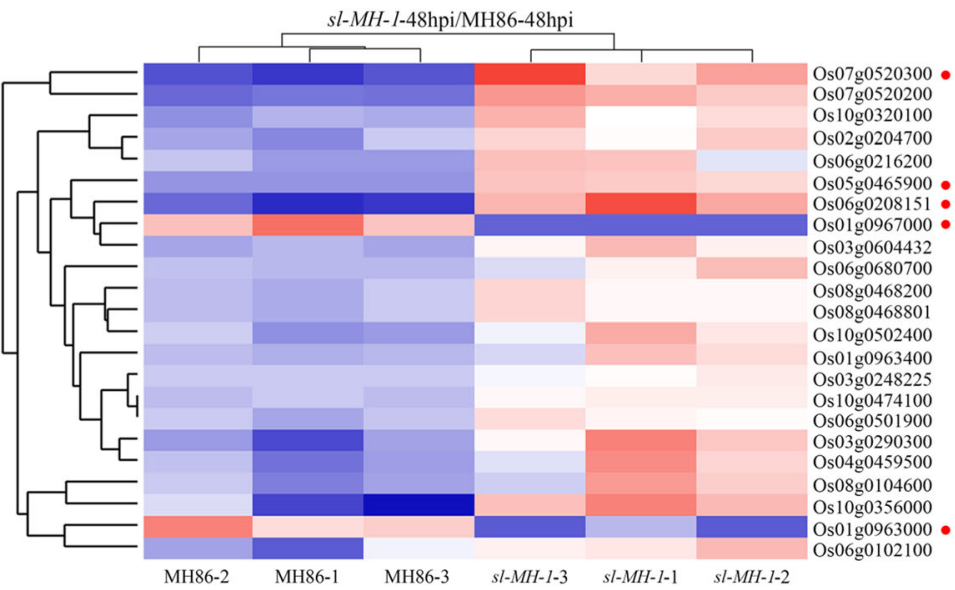

Fig. 7 Transcritome analysis in MH86 and sl-MH-1 plants with and without blast fungus inoculation. a Gene Ontology enrichment analysis showed the changed transcript profiles in various biological processes of sl-MH-1, compared with MH86, after $P$. oryzae inoculation. $\mathbf{b}$ Heat maps of the significant differentially expressed genes (DEGs) in the redox pathway from sl-MH-1-mock compared with MH86-mock and sl-MH-1-48hpi compared with MH86-48hpi. The red dots indicate the genes with similar trend of transcriptional change in sl-MH-1-mock/MH86-mock and sl-MH1-48hpi/MH86-48hpi. c The relative transcriptional levels of SL in NPB and NPB-Pizt plants before and after inoculation with P. oryzae isolate KJ201. $U B Q$ was used as the internal control. Bars represent mean values \pm SD $(n=3)$. Statistically significant difference was indicated by ${ }^{* *}(p<0.01$, Student's $t$-test). The data are from one of three independent experiments performed with similar results 
MH86-mock and sl-MH-1-48hpi/MH86-48hpi (Fig. 7b, indicated by red dots), suggesting their transcriptional changes caused by defective SL was not affected by blast fungus infection. Among them, Os07g0520300 encodes phytohormone-related protein of cytochrome P450 family, whose transcriptional level was significantly increased in sl-MH-1; Os01g0963000 and Os01g0967000 were the only two down-regulated redox genes in $s l-M H-1$ after inoculation, which encodes cationic peroxidase SPC4 and LSM domain containing protein, respectively. SPC4 potentially involved in ROS generation in rice [20].

Among the other eighteen redox-related DEGs in sl48hpi/MH86-48hpi, all of which were up-regulated in $s l$ MH-1 mutant, five genes, Os06g0102100, Os02g0204700, Os06g0680700, Os10g0320100 and Os06g0501900, encode cytochrome P450 family proteins; Os10g0356000 and Os10g0502400 mainly expressed in photosynthetic system, the later was regulated by phytochromes and cryptochromes under different light conditions [21, 22]; Os08g0104600 encodes an chloroplast-located ferredoxin, involved in transferring electron from photosystem I to $\mathrm{NADP}^{+}$and the various acceptor systems of metabolic processes [23]; Os03g0290300 and Os06g0216200 (OsOPR2) encodes a $\omega-3$ fatty acid desaturase and a OPR isozyme, respectively, which are involved in JA biosynthesis [24-26]; Os04g0459500 encodes a glyceraldehyde-3-phosphate dehydrogenase (GAPDH), which translocates to the nucleus during apoptosis and influences cytotoxicity [27]. To confirm the above RNA-seq results, we performed quantitative real-time PCR (qRT-PCR) analysis of the key genes in redox pathway, including Os01g0963000, Os08g0104600 and Os03g0290300, in MH86 and sl-MH-3 before and at $48 \mathrm{~h}$ after $P$. oryzae inoculation. As shown in Fig. S6, the transcriptional changes of these genes in $s l-M H-3$ are similar with those in $s l-M H-1$ transcritome data.

In addition, we investigated $S L$ transcription in the $j a$ ponica rice cultivar Nipponbare (NPB) and Piz-t-transgenic NPB (NPB-Pizt) plants before and after inoculation with the blast fungal isolate $\mathrm{KJ} 201$, which is avirulent to Piz-t $[19,28]$. As shown in Fig. 7c, no significant difference of $S L$ transcriptional levels in NPB and NPB-Pizt was detected before or at $48 \mathrm{~h}$ after inoculation. With disease development, $S L$ transcription in NPB was significantly upregulated at $72 \mathrm{hpi}$, when it was not obviously altered in NPB-Pizt (Fig. 7c). These results suggest that successful $P$. oryzae infection could up-regulate $S L$ expression in rice.

\section{Discussion}

\section{Sekiguchi lesion is resulted from high accumulation of endogenous ROS}

The biological functions of serotonin in rice are mainly explored via characterizing phenotypes of the sekiguchi lesion mutant, as SL is required for converting tryptamine to serotonin. In this study, we identified the mutants of $S L$ gene in MH86 background, which also exhibit sekiguchi lesion under normal growth condition and markedly decreased serotonin content. $s l-M H-1 \mathrm{mu}-$ tant accumulates high level of $\mathrm{H}_{2} \mathrm{O}_{2}$ at its lesion sites and is more sensitive to exogenous $\mathrm{H}_{2} \mathrm{O}_{2}$ treatment. Furthermore, $\mathrm{VC}$ treatment could suppress the lesion formation on sl-MH-1 leaves, and the similar result has been described in the $s l$ mutant [29]. These results suggest that when SL is defective, high endogenous oxidation level leads to cell death occurrence, which is consistent with the role of serotonin as an effective internal ROS scavenger [30]. Meanwhile, when $S L$ is mutated, the $\mathrm{H}_{2} \mathrm{O}_{2}$ generated from oxidation of the excessive tryptamine by monoamine oxidase could also contribute to the lesion formation [29]. Excessive ROS production could contribute to or execute PCD by rapidly oxidizing and damaging cellular components, including proteins, nucleic acids and lipids. ROS-induced PCD has been described as a mechanism of photooxidative damage in plants during photosynthesis [31]. It has been reported that the most effective wavelength for inducing typical sekiguchi lesion was $400-700 \mathrm{~nm}$; whereas in the dark, only brown spots and necrotic spot lesions formed on the $s l$ leaves [32]. In addition, sekiguchi lesion could be significantly inhibited by the photosynthetic inhibitor [33]. Therefore, we speculate that under normal growth condition, serotonin is essential for scavenging endogenous oxygen radicals produced during photosynthesis, and thus protecting rice from oxidative damage.

\section{SL negatively regulates rice resistance to biotrophic pathogens}

In this study, we found that $s l-M H$ mutants displayed enhanced resistance to both blast fungus and blight bacteria. $s l-M H-1$ formed the typical lesion surrounding the infection sites, which could efficiently inhibit the pathogen extension. Moreover, the transcriptional level of $S L$ was up-regulated in NPB but not NPB-Pizt at $72 \mathrm{hpi}$ with the blast fungal isolate avirulent to Piz-t, suggesting that a compatible interaction between rice and $P$. oryzae could induce $S L$ expression. It has been supposed that $72 \mathrm{hpi}$ is the stage when $P$. oryzae transits from the biotrophic to the necrotrophic phase during its disease cycle [34]. Therefore, the up-regulation of $S L$ expression at this time point may be required for alleviating high oxidative stress caused by host cell collapse upon entering necrotrophic phase. Similarly, serotonin was indicated to function in protecting uninfected tissues from oxidative damage caused by the HR [35].

Our data also showed that $s l-M H-1$ mutant displayed more robust PTI responses, including PAMPs-induced ROS burst and callose deposition. High oxidative level in plant cells plays multifaceted signaling roles in mediating 
the establishment of immune responses [36]. For example, ROS could stimulate a rapid $\mathrm{Ca}^{2+}$ influx upon elicitation [37]. All the plant plasma membrane-localized respiratory burst oxydase homologs (Rbohs), which are essential for apoplastic ROS production [38], contain the $\mathrm{Ca}^{2+}$ - binding EF hand motifs at their $\mathrm{N}$ terminus, implying that ROS production and $\mathrm{Ca}^{2+}$ influx co-regulate each other [39]. Except that, ROS directly mediates the cross-linking of plant cell wall components to strength the structural barriers against pathogens [40]. Moreover, ROS could also contribute to the activation of plant immune responses by inducing changes in gene expression [41]. Thus, we suppose that the enhanced PTI responses of the $s l-M H$ mutant may also be resulted from its high endogenous ROS accumulation.

In contrast to its enhanced resistance to $P$. oryzae and $X o o$, the $s l$ mutant was reported to be more susceptible to $B$. oryzae [12]. The divergent pathogenic phenotypes on $s l$ mutants could be resulted from the different trophic types of these pathogens. B. oryzae is a necrotrophic pathogen, which takes nutrition from dead host cells. In this case, the spontaneous cell death of $s l \mathrm{mu}-$ tants could be a favorable term for $B$. oryzae growth. However, P. oryzae and Xoo are hemibiotrophic and biotrophic pathogens, respectively, to whose colonization, lesion of $s l$ mutants could set a limitation.

\section{SL functions in suppressing accumulation of the defense hormones}

SA and JA are the important phytohormones that trigger and mediate a series of defense responses. In Arabidopsis, JA is involved in resistance against necrotrophic pathogens and herbivorous insects [42], whereas SA contributes to defense responses against biotrophic pathogens [43]. And the SA and JA-mediated immune pathways in Arabidopsis are always antagonistic to each other. Although the role of SA in defense is conserved in rice, the SA content is less essential for inducing rice resistance, as rice plants usually accumulate high level of SA during normal growth $[44,45]$. Interestingly, SA could also contribute to rice defense against brown planthopper [46]. Furthermore, in contrast to the finding in Arabidopsis, JA in rice positively regulates immune responses against (hemi)biotrophic pathogens $[47,48]$.

Our results indicated that SA constantly accumulates in $s l-M H-1$ mutant to a level about twice as high as that in the wild type, and the contents of JA and JA-Ile are dramatically increased in $s l-M H-1$ when the lesions appear. Considering that SA in rice is essential for modulating redox balance and scavenging the endogenous ROS [49], we speculate that the high SA accumulation in sl-MH-1 may be employed to alleviate its internal oxidative stress. JA is a lipid-derived hormone, whose chloroplastic intermediate, cis-(1)-12-oxophytodienoic acid
(OPDA), is derived from oxidatively modified polyunsaturated fatty acids [50]. Thus the increased JA and JA-Ile contents in $s l-M H-1$ may also be caused by the raised oxidative level, as high levels of JA and its derivant were detected in Arabidopsis when more ROS was accumulated by deletion of $\mathrm{Fd} 2$, the major ferredoxin in chloroplasts [23]. On the other hand, Os03g0290300, encoding a $\omega-3$ fatty acid desaturase that is involved in the synthesis of unsaturated fatty acids serving as JA precursor, was significantly up-regulated in $s l-M H$ mutants, which could contribute to JA and JA-Ile production as well.

\section{Conclusions}

In this study, we identified $s l$ mutants in MH86 background $(s l-M H)$ and revealed the roles of SL in rice innate immunity. Our results suggest the following conclusions: (i) the $s l-M H$ mutant is more sensitive to exogenous ROS stress, its lesions formation is mainly resulted from excessive accumulation of internal ROS; (ii) SL negatively regulates rice defense against blast fungus and blight bacteria via compromising the PTI responses and suppressing the defense hormones accumulation; (iii) loss function of SL dramatically alters transcription of the genes involved in redox pathway after inoculation with $P$. oryzae; (iv) success $P$. oryzae infection upregulates the transcriptional level of $S L$ in rice. Taken together, our study decipher the negative roles of SL in rice defense against (hemi)biotrophic pathogens.

\section{Methods}

\section{Plant materials and blast isolates}

The indica rice restore line Minghui 86 was originally bred at Institute of Rice, Fujian Academy of Agricultural Sciences, Fuzhou, China. The sl-MH-1 mutant was generated from the tissue culture-induced mutation of MH86 as described previously [15]. The $s l-M H-2$ and $s l$ $M H-3$ mutants in this study were generated from ${ }^{60} \mathrm{Co}$ $\sim \gamma$-ray irradiation of MH86 by us. The rice cultivar Nipponbare, its transgenic line carrying Piz-t (NPB-Pizt) and the $P$. oryzae isolate KJ201 were originally obtained from Dr. Guo-Liang Wang's laboratory (Department of Plant Pathology, Ohio State University, Columbus, Ohio). The P. oryzae isolate FJ-1 (virulent to Minghui86) was provided by Dewei Yang (Institute of Rice, Fujian Academy of Agricultural Sciences, Fuzhou, China). The Xoo strain race 6 was provided by Dr. Dingzhong Tang (Plant Immunity Center, Fujian Agriculture and Forestry University, Fuzhou, China). For the $\mathrm{H}_{2} \mathrm{O}_{2}$ treatment, $P$. oryzae inoculation, PTI responses detection and defense hormones measurement assays, the plants were germinated and grown in a growth chamber at $28{ }^{\circ} \mathrm{C}$ under a 12-h light $\left(600-800 \mu \mathrm{mol} / \mathrm{m}^{2} . \mathrm{s}\right) / 12$-h dark cycle. For the metabolites measurement, lesion staining, $\mathrm{VC}$ treatment 
and $\mathrm{Xoo}$ inoculation assays, the plants were grown in a humidity-controlled greenhouse under natural conditions during summer season.

\section{Blast fungus and blight bacteria inoculation}

$P$. oryzae and Xoo inoculations were carried out in growth chamber and greenhouse, respectively. P. oryzae isolate FJ-1 was cultured on CMII medium for 2 weeks under light for sporulation. Then a conidial suspension with $3 \times 10^{5}$ spores $/ \mathrm{ml}$ was sprayed on 3-week-old rice leaves. After inoculation, the seedlings were maintained in the dark for $24 \mathrm{~h}$ at $28^{\circ} \mathrm{C}$ with high humidity, then transferred into the growth chamber for $5 \sim 7$ days to evaluate their disease symptom. Four-week-old rice plants were used to perform punch inoculation as previously described [19], and a $10-\mu \mathrm{l}$ volume of a spore suspension $\left(2 \times 10^{5}\right.$ spores $\left./ \mathrm{ml}\right)$ was applied. Investigation of the fungal biomass in infected rice leaf tissue was carried out as the method described previously [51]. Two punched leaves from one single plant were collected as one biological replicate for the statistic analysis. For leaf sheath inoculation, conidial suspension was injected to the detached sheath cavum of 21-day-old rice plants. Then, the inoculated sheath was kept in an incubator with $80 \%$ humidity for the indicated time. Prior to microscopy observing, the surface cell of inner sheath was peeled and made as a slide sample. Inoculation with Xoo was conducted during the tilling stage by the leaf clipping method [52]. The bacterial suspension with optical density at $600 \mathrm{~nm}\left(\mathrm{OD}_{600}\right)=0.5$ was used to inoculate rice leaves as follows: scissor was dipped in the bacterial suspension and then used to cut the tip of a rice leaf. The inoculated plants were kept in the greenhouse for 14 days before disease symptom analysis. The blight lesion length and bacterial population accumulated in the infected leaves were evaluated as reported previously [53].

\section{VC treatment}

VC $(0.1 \%$, diluted with distilled water) was sprayed onto the 3-week-old rice seedlings three times per day (at 9: $00,12: 00$ and 15:00) for 10 days. Water spraying was used as control.

\section{Analysis of metabolites content}

$1000 \mu \mathrm{l}$ of precooled extract solution (acetonitrile:methanol:water, 2:2:1) was added to each $50 \mathrm{mg}$ ground leave samples of MH86 and sl-MH-1. Metabolites extraction was performed as described previously [54] with minor modification as below. After homogenate-sonicate circles and centrifugation, $100 \mu \mathrm{l}$ of the supernatant was transferred and dried under a nitrogen flow, then $100 \mu \mathrm{l}$ of $10 \%$ methanol was used to reconstitute the residual. Following centrifugation, $80 \mu \mathrm{l}$ of the supernatant was transferred into an auto-sampler vial for UHPLC-MS/
MS analysis. Preparing the standard solution and the UHPLC separation was carried out as described before [54]. L-2-Chloro-phenylalanine was used as the internal standard (IS) at a concentration of $200 \mathrm{nmol} / \mathrm{l}$. Mobile phase $\mathrm{A}$ was $0.1 \%$ acetic acid in water, and mobile phase $\mathrm{B}$ was methanol. The elution time and gradient for UHPLC separation and quantification analysis of the metabolites content were shown in Table S3. Reproducibility was assessed using five biological replicates in each experiment.

\section{SA and JA contents measurement}

For measuring the defense hormones contents, the leaves from three 6-week-old MH86, sl-MH-1 or sl-MH3 plants were harvested as one biological replicate; the leaves from eight 3-week-old MH86 or sl-MH-1 plants were harvested as one biological replicate. The method of measurement is as previously described [55].

\section{DAB and Trypan blue staining}

The leaf samples of 8-week-old MH86 and sl-MH-1

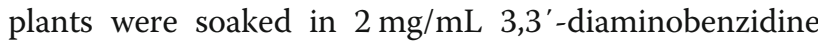
(DAB) $\quad(2 \mathrm{mg} / \mathrm{mL} \quad \mathrm{DAB}, 0.05 \%$ Tween $20,10 \mathrm{mM}$ $\mathrm{Na}_{2} \mathrm{HPO}_{4}$ ) for $4 \sim 5 \mathrm{~h}$ then transferred to the destaining solution (ethanol:lactic acid: Glycerol = 3:1:1). After heating to $90-95^{\circ} \mathrm{C}$ for $15 \mathrm{~min}$, the leaves were transferred to a fresh destaining solution and shaked gently overnight. Trypan blue staining was carried out as previously described [56].

\section{Callose deposition}

For observing callose deposition, the rice leaves from $7-$ day-old seedling were treat with chitin (hexa- $\mathrm{N}$-acetylchitohexaose). The assay was carried out as previously described [57]. Finally the leaves were observed under UV light (340 to $380 \mathrm{~nm}$; Zeiss LSM880).

\section{Seedling treated with $\mathrm{H}_{2} \mathrm{O}_{2}$}

The dehusking rice seeds were sterilized with $75 \%$ ethanol for $2 \mathrm{~min}$ and $3 \%$ sodium hypochlorite for $30 \mathrm{~min}$, then germinated on $1 / 2$ MS medium with or without $0.02 \% \mathrm{H}_{2} \mathrm{O}_{2}$. The plates were put in a $28^{\circ} \mathrm{C}$ growth chamber with a 12-h light/12-h dark cycle for 7 days. Then the seedlings were imaged for analyzing sensitivity to $\mathrm{H}_{2} \mathrm{O}_{2}$ treatment.

\section{ROS burst detection}

Leaf discs from 7-day-old rice seedlings were floated on sterilized water overnight, then put into $100 \mathrm{ul}$ reaction solution (20 uM luminal and $2.5 \mu \mathrm{g} / \mathrm{mL}$ peroxidase) containing $500 \mathrm{nM}$ flg22 or $400 \mathrm{nM}$ chitin to immediately test the ROS burst. The luminescence was measured by Berthold Mithras luminometer every 1-2 min for $1 \mathrm{~h}$. Each data point represented eight replicates. 


\section{RNA-seq sequencing and qRT-PCR analysis}

The total RNA from MH86 and sl-MH-1 leaves before and after blast fungus spraying inoculation was extracted using the RNAeasy kit (Qiagen, Germany) and treated with an RNase-Free DNAse Set (Qiagen, Germany), according to the manufacturer's instructions. The RNA quality, library construction and size were assayed using a 2100 Bioanalyzer system (Agilent, USA). The libraries were synthesized using the TruSeq RNA Sample Preparation v2 kit (Illumina, USA). Total RNA from each treatment was pooled and then two libraries were constructed and used for sequencing. The samples were run in the NovaSeq system and raw sequences of paired 150-bp were obtained.

The analysis below was run on Majorbio (https://www. i-sanger.com). Raw reads were evaluated using fastx toolkit_0.0.14. SeqPrep and Sickle software were used for clean data. TopHat2 was used to map reads individually for each biological replicate in MH86 or $s l-M H-1$ against $O$. sativa japonica cultivar Nipponbare sequence according to MSU 7.0. Cufflinks was used for assembly into transcripts with reference annotation to guide assembly [58]. All the produres were completed according to the settled parameter. The significant differentially expressed loci (SDEL) in $s l-M H-1$ mutant compared to MH86 were identified after applying multiple corrections (FDR adjusted $p \leq 0.05$ ). The SDEL with fold change $\geq 2$ or $\leq 0.5$ were used for further analysis. Functional enrichment analysis (FEA) was performed on SDEL in sl-MH-1 compared to MH86. Functional annotation and expression correlation analysis were conducted on the gene set related to oxidation-reduction biological process. Heat map was generated using cluster analysis.

qRT-PCR analysis was carried out as previously described [23]. The primers used for qRT-PCR in this study are listed in Table S4.

\section{Supplementary Information}

The online version contains supplementary material available at https://doi. org/10.1186/s12870-020-02724-6.

\section{Additional file 1: Fig. S1. Lesion phenotype of the allelic sl-MH mutants The leaves of $\mathrm{MH} 86$, sl-MH-1, sl-MH-2 and sl-MH-3 were photographed after grown in greenhouse for 6 weeks.}

Additional file 2: Fig. S2. Schematic representation of $S L$ gene structure and the mutation sites. Black boxes and lines indicate exons and introns, respectively, and untranslated regions are shown in grey boxes. The arrows indicate the mutation sites of the allelic sl-MH mutants.

Additional file 3: Fig. S3. The content of L-glutamine in MH86 and st$\mathrm{MH}-1$. The levels of $\mathrm{L}$-glutamine in 8-week-old MH86 and sl-MH-1 plants were measured by UPLC. Bars represent mean values \pm SD from five biological replicates. Statistically significant difference was indicated by ** ( $p<0.01$, Student's $t$-test).

Additional file 4: Fig. S4. Resistance of $s /-M H-3$ to P. oryzae and Xoo. a. 3-week-old MH86 and sI-MH-3 seedlings were inoculated with P. oryzae conidia by spraying and the diseased leaves were imaged at $7 \mathrm{dpi}$. b. Punch inoculation with P. oryzae conidia was performed on the leaves of 4-week-old MH86 and sl-MH-3 plants. The diseased leaves were photographed at $9 \mathrm{dpi}$ (left), and the fungal biomass was measured (right). Bars represent mean values \pm SD from three biological replicates. Statistically significant difference was indicated by ** $(p<0.01$, Student's $t$-test). c. MH86 and sl-MH-3 plants were inoculated with Xoo. The infected leaves from three independent $\mathrm{MH} 86$ or sl-MH-3 plants were imaged at $14 \mathrm{dpi}$. d. Blight lesion length on SI-MH-3 and MH86 leaves were measured (left), bars represent mean values $\pm \mathrm{SD}(n=6$, from three independent plants; * means $p<0.05$ by Student's $t$-test). Blight bacterial populations were counted $14 \mathrm{dpi}$ with bars representing mean values \pm SD from three independent MH86 or sl-MH-3 plants (right), ** means $p<0.01$ by Student's $t$-test. e. Callose deposition on MH86 and sl-MH-3 leaves after chitin treatment was imaged with a microscope under UV light (left). Number of the callose deposition per view was counted (right). Bars represent mean values and SD $(n=5)$. Statistically significant difference was indicated by ** $(p<0.01$, Student's $t$-test).

Additional file 5: Fig. S5. Contents of defense hormones in 3-week-old MH86 and sl-MH-1 plants. The resting levels of SA, JA and JA-lle in 3week-old MH86 and sl-MH-1 leaves were measured by UPLC. Bars represent mean values \pm SD from three biological replicates. Statistically significant difference was indicated by ${ }^{* *}(p<0.01$, Student's $t$-test).

Additional file 6: Fig. S6. qRT-PCR analysis of the representative genes of redox pathway in MH86 and sl-MH-3. The transcriptional levels of Os01g0963000, Os08g0104600 and Os03g0290300 were determined in MH86 and sl-MH-3 at 48 hpi with P. oryzae (water spraying was employed as mock treatment). UBQ was used as the internal control. Bars represent mean values \pm SD $(n=3)$. Statistically significant difference was indicated by ** ( $p<0.01$, Student's $t$-test).

Additional file 7: Table S1. Significant differentially expressed genes in sl-MH-1-mock/MH86-mock.

Additional file 8: Table S2. Significant differentially expressed genes in sl-MH-1-48hpi/MH86-48hpi.

Additional file 9: Table S3. Quantification of serotonin, tryptamine, $L-$ trytophan and L-glutamine by UHPLC-MS.

Additional file 10: Table S4. Sequences of the GRT-PCR primers in this study.

\section{Abbreviations}

PCD: Programmed cell death; Vc: Vitamin C; PAMPs: Pathogen-associated molecular patterns; PTI: PAMPs-triggered immunity; ROS: Reactive oxygen species; P. oryzae: Pyricularia oryzae; Xoo: Xanthomonas oryzae pv. Oryzae; $B$ : oryzae: Bipolaris oryzae; redox: Reduction-oxidation; ETI: Effector-triggered immunity; HR: Hypersensitive response; sl: sekiguchi lesion; DAB: 3,3diaminobenzidine tetrahydrochloride; MS: Murashige and Skoog; SA: Salicylic acid; JA: Jasmonic acid; JA-lle: JA-isoleucine; hpi: Hours post inoculation; SDEL: Significant differentially expressed loci; DEGs: Differentially expressed genes; RNA-Seq: RNA-sequencing; qRT-PCR: quantitative real-time polymerase chain reaction

\section{Acknowledgements}

We thank Dr. Songbiao Chen at Institute of Oceanography, Minjiang University, Fuzhou, China for sharing sl-MH-1 seeds. Dr. Dingzhong Tang at Plant Immunity Center, Fujian Agriculture and Forestry University, Fuzhou, China for providing the Xoo strain race 6, Dewei Yang at Institute of Rice, Fujian Academy of Agricultural Sciences, Fuzhou, China for providing the $P$. oryzae isolate FJ-1, and Jinfang Chu at Institute of Genetics and Developmental Biology, Chinese Academy of Sciences, Beijing, China for helping us to measure and analyze contents of the defense hormones.

\section{Authors' contributions}

MW and DT conceived and designed the project. FY, YN and DT performed the experiments. DT, FY, YN, YL, ZC and GL analyzed the data. MW, DT, QL and FW wrote and edited the manuscript. All the authors have read and approved the final manuscript. 


\section{Funding}

This work was supported by the Natural Science Foundation of Fujian Province (Number: 2014 J01102), Youth Program of National Natural Science Foundation of China (Number: 31301654), China Postdoctoral Science Foundation (Number: 2019 M662919) and National R\&D Project of Transgenic Crops of Ministry of Science and Technology of China (Number: 2017ZX08001-001).

\section{Availability of data and materials}

All data sustaining the results in this study are included in this manuscript or its supplementary information files. The datasets analyzed during the current study are available from the corresponding author on reasonable request. The raw sequencing data reported in this article have been deposited in NCBI SRA database, under accession number PRJNA634690, which are publicly accessible at http://www.ncbi.nlm.nih.gov/bioproject/634690.

\section{Ethics approval and consent to participate}

Not applicable.

\section{Consent for publication}

Not applicable.

\section{Competing interests}

The authors declare no conflicts of interest.

\section{Author details}

${ }^{1}$ Biotechnology Research Institute, Fujian Key Laboratory of Genetic Engineering for Agriculture, Fujian Academy of Agricultural Sciences, Fuzhou 350003, Fujian, China. ${ }^{2}$ State Key Laboratory of Ecological Pest Control for Fujian and Taiwan Crops, College of Life Science, Fujian Agriculture and Forestry University, Fuzhou 350002, Fujian, China. ${ }^{3}$ Key Laboratory of Ministry of Education for Genetics, Breeding and Multiple Utilization of Crops, College of Agriculture, Fujian Agriculture and Forestry University, Fuzhou 350002, Fujian, China. ${ }^{4}$ Ministry of Education Key Laboratory of Agriculture Biodiversity for Plant Disease Management, Yunnan Agricultural University, Kunming 650201, China. ${ }^{5}$ Fujian University Key Laboratory for Plant-Microbe Interaction, Fujian Agriculture and Forestry University, Fuzhou 350002, Fujian, China.

Received: 22 April 2020 Accepted: 26 October 2020 Published online: 04 November 2020

\section{References}

1. Jones JDG, Dangl JL. The plant immune system. Nature. 2006;444(7117): 323-9.

2. Tang D, Wang G, Zhoud J-M. Receptor kinases in plant-pathogen interactions: more than pattern recognition. Plant Cell. 2017;29:618-37.

3. Peng Y, Wersch RV, Zhang Y. Convergent and divergent signaling in PAMPtriggered immunity and effector-triggered immunity. Mol Plant-Microbe Interact. 2017:31(4):403-9.

4. Hofius D, Tsitsigiannis DI, Jones JDG, Mundy J. Inducible cell death in plant immunity. Semin Cancer Biol. 2007;17(2):166-87.

5. Spoel SH, Loake GJ. Redox-based protein modifications: the missing link in plant immune signalling. Curr Opin Plant Biol. 2011;14(4):358-64.

6. Coll NS, Epple P, Dangl JL. Programmed cell death in the plant immune system. Cell Death Differ. 2011;18(8):1247-56.

7. Veenstra-Vanderweele J, Anderson GM, Cook EH. Pharmacogenetics and the serotonin system: initial studies and future directions. Eur J Pharmacol. 2000; 410(2):165-81.

8. Bowden K, Brown BG, Batty JE. 5-Hydroxytryptamine: its occurrence in cowhage (Mucuna pruriens). Nature. 1954;174:925-6.

9. Ramakrishna A, Giridhar P, Ravishankar GA. Phytoserotonin: a review. Plant Signal Behav. 2011;6(6):800-9.

10. Schröder P, Abele C, Gohr P, Stuhlfauth-Roisch U, Grosse W. Latest on enzymology of serotonin biosynthesis in walnut seeds. Adv Exp Med Biol. 1999:467:637-44.

11. Huether G, Fettkötter I, Keilhoff G, Wolf G. Serotonin acts as a radical scavenger and is oxidized to a dimer during the respiratory burst of activated microglia. J Neurochem. 2010;69(5):2096-101.

12. Ishihara A, Hashimoto Y, Tanaka C, Dubouzet JG, Nakao T, Matsuda F, Nishioka T, Miyagawa H, Wakasa K. The tryptophan pathway is involved in the defense responses of rice against pathogenic infection via serotonin production. Plant J. 2008;54(3):481-95.

13. Liu W, Liu J, Triplett L, Leach JE, Wang GL. Novel insights into rice innate immunity against bacterial and fungal pathogens. Annu Rev Phytopathol. 2014;52(1):213-41.

14. Fujiwara T, Maisonneuve $\mathrm{S}$, Isshiki M, Mizutani M, Chen L, Wong HL, Kawasaki T, Shimamoto K. Sekiguchi lesion gene encodes a cytochrome P450 monooxygenase that catalyzes conversion of tryptamine to serotonin in rice. J Biol Chem. 2010;285(15):11308-13.

15. Gao X, Chen Z, Song Y, Chen Z, Tian D, Lin Y, Yang S, Chen S, Wang F. Identification and gene mapping of a runaway cell death mutant rcd1 in Rice. Mol Plant Breed. 2015;13:1433-40.

16. Pavet V, Olmos E, Kiddle G, Mowla S, Kumar S, Antoniw J. Alvarez MaE, foyer $\mathrm{CH}$ : ascorbic acid deficiency activates cell death and disease resistance responses in Arabidopsis. Plant Physiol. 2005:139(3):1291-303.

17. Wang R, Liu S, Zhou F, Ding C. Exogenous ascorbic acid and glutathione alleviate oxidative stress induced by salt stress in the chloroplasts of Oryza sativa L. Z Naturforsch C. 2014;69:226-36.

18. Hasegawa M, Mitsuhara I, Seo S, Imai T, Koga J, Okada K, Yamane H, Ohashi Y. Phytoalexin accumulation in the interaction between Rice and the blast fungus. Mol Plant-Microbe Interact. 2010;23(8):1000-11.

19. Park CH, Shirsekar G, Bellizzi M, Chen S, Songkumarn P, Xie X, Shi X, Ning Y, Zhou B, Suttiviriya P, et al. The E3 ligase APIP10 connects the effector AvrPiz-t to the NLR receptor Piz-t in Rice. PLoS Pathog. 2016;12(3):e1005529.

20. Liu X, Williams CE, Nemacheck JA, Wang H, Subramanyam S, Zheng C, Chen $\mathrm{M}-\mathrm{S}$. Reactive oxygen species are involved in plant defense against a gall midge. Plant Physiol. 2010;152(2):985-99.

21. Mccormac AC, Fischer A, Kumar AM, Söll D, Terry MJ. Regulation of HEMA1 expression by phytochrome and a plastid signal during de-etiolation in Arabidopsis Thaliana. Plant J. 2001;25(5):549-61.

22. Tanaka R, Tanaka A. Chlorophyll cycle regulates the construction and destruction of the light-harvesting complexes. Biochimica et Biophysica Acta (BBA)/Bioenergetics. 2011;1807(8):968-76.

23. Wang M, Rui L, Yan H, Shi H, Zhao W, Lin JE, Zhang K, Blakeslee JJ, Mackey $\mathrm{D}$, Tang $\mathrm{D}$, et al. The major leaf ferredoxin $\mathrm{Fd} 2$ regulates plant innate immunity in Arabidopsis. Mol Plant Pathol. 2018;19(6):1377-90.

24. Sobajima H, Takeda M, Sugimori M, Kobashi N, Kiribuchi K, Cho E-M, Akimoto C, Yamaguchi T, Minami E, Shibuya N, et al. Cloning and characterization of a jasmonic acid-responsive gene encoding 12oxophytodienoic acid reductase in suspension-cultured rice cells. Planta. 2003;216(4):692-8

25. Taki N, Sasaki-Sekimoto Y, Obayashi T, Kikuta A, Kobayashi K, Ainai T, Yagi K, Sakurai N, Suzuki H, Masuda T, et al. 12-oxo-phytodienoic acid triggers expression of a distinct set of genes and plays a role in wound-induced gene expression in Arabidopsis. Plant Physiol. 2005;139(3):1268-83.

26. Goepfert $S$, Poirier Y. $\beta$-Oxidation in fatty acid degradation and beyond. Curr Opin Plant Biol. 2007;10(3):245-51.

27. Hara MR, Agrawal N, Kim SF, Cascio MB, Fujimuro M, Ozeki Y, Takahashi M, Cheah JH, Tankou SK, Hester LD, et al. S-nitrosylated GAPDH initiates apoptotic cell death by nuclear translocation following Siah1 binding. Nat Cell Biol. 2005;7(7):665-74

28. Zhou B, Qu S, Liu G, Dolan M, Sakai H, Lu G, Bellizzi M, Wang GL. The eight amino-acid differences within three leucine-rich repeats between $\mathrm{Pi} 2$ and Piz-t resistance proteins determine the resistance specificity to Magnaporthe grisea. Mol Plant-Microbe Interact. 2006;19(11):1216-28.

29. Ueno M, Shibata H, Kihara J, Honda Y, Arase S. Increased tryptophan decarboxylase and monoamine oxidase activities induce Sekiguchi lesion formation in rice infected with Magnaporthe grisea. Plant J. 2003; 36(2):215-28

30. Huether $\mathrm{G}$, Fettkötter I, Keilhoff $\mathrm{G}$, Wolf $\mathrm{G}$. Serotonin acts as a radical scavenger and is oxidized to a dimer during the respiratory burst of activated microglia\&nbsp. J Neurochem. 2002;69(5):2096-101.

31. Triantaphylides C, Krischke M, Hoeberichts FA, Ksas B, Gresser G, Havaux M, Van Breusegem F, Mueller MJ. Singlet oxygen is the major reactive oxygen species involved in Photooxidative damage to plants. Plant Physiol. 2008; 148(2):960-8.

32. Arase S, Fukuyama R, Honda Y. Light-dependent induction of Sekiguchi lesion formation by Bipolaris oryzae in rice cv. Sekiguchi-asahi. J Phytopathol. 2000;148(4):193-6. 
33. ledome M, Arase S, Honda Y, Nozu M. Light-dependent necrosis formation by Magnaporthe grisea toxin(s) in Rice cv. Sekiguchi-asahi. J Phytopathol. 2008;143:325-8.

34. Mathioni SM, Beló A, Rizzo CJ, Dean RA, Donofrio NM. Transcriptome profiling of the rice blast fungus during invasive plant infection and in vitro stresses. BMC Genomics. 2011:12:49.

35. Hayashi K, Fujita Y, Ashizawa T, Suzuki F, Nagamura Y, Hayano-Saito Y. Serotonin attenuates biotic stress and leads to lesion browning caused by a hypersensitive response to Magnaporthe oryzae penetration in rice. Plant Cell Mol Biol. 2016;85(1):46-56.

36. Lehmann S, Serrano M, L'Haridon F, Tjamos SE, Metraux J-P. Reactive oxygen species and plant resistance to fungal pathogens. Phytochemistry. 2014;112:54-62

37. Levine A, Pennell RI, Alvarez ME, Palmer R, Lamb C. Calcium-mediated apoptosis in a plant hypersensitive disease resistance response. Curr Biol. 1996;6(4):427-37.

38. Torres MA, Dangl JL, Jones JDG. Arabidopsis gp91phox homologues AtrbohD and AtrbohF are required for accumulation of reactive oxygen intermediates in the plant defense response. Proc Natl Acad Sci. 2002;99(1): 517-22

39. Torres MA, Dangl JL. Functions of the respiratory burst oxidase in biotic interactions, abiotic stress and development. Curr Opin Plant Biol. 2005;8(4): 397-403.

40. Almagro L, Gomez Ros LV, Belchi-Navarro S, Bru R, Ros Barcelo A, Pedreno MA. Class III peroxidases in plant defence reactions. J Exp Bot. 2009;60(2): 377-90.

41. Kotchoni SO, Gachomo EW. The reactive oxygen species network pathways: an essential prerequisite for perception of pathogen attack and the acquired disease resistance in plants. J Biosci. 2006;31(3):389-404.

42. Glazebrook J. Contrasting mechanisms of defense against biotrophic and necrotrophic pathogens. Annu Rev Phytopathol. 2005;43:205-27.

43. Vlot AC, Dempsey DMA, Klessig DF. Salicylic acid, a multifaceted hormone to combat disease. Annu Rev Phytopathol. 2009;47:177-206.

44. Yang D-L, Yang Y, He Z. Roles of plant hormones and their interplay in Rice immunity. Mol Plant. 2013;6:675-85

45. Silverman P, Seskar M, Kanter D, Schweizer P, Métraux J-P, Raskin I. Salicylic acid in Rice: biosynthesis, conjugation and possible role. Plant Physiol. 1995; 108:633-9.

46. Lu HP, Luo T, Fu HW, Wang L, Tan Y-y, Huang J-z, Wang Q, Ye G-y, Gatehouse A, Lou Y-g, et al. Resistance of rice to insect pests mediated by suppression of serotonin biosynthesis. Nature Plants. 2018;4(6):338-44.

47. Yamada S, Kano A, Tamaoki D, Miyamoto A, Shishido H, Miyoshi S, Taniguchi S, Akimitsu K, Gomi K. Involvement of OsJAZ8 in Jasmonateinduced resistance to bacterial blight in Rice. Plant Cell Physiol. 2012;53(12): 2060-72.

48. Mei C, Qi M, Sheng G, Yang Y. Inducible overexpression of a rice allene oxide synthase gene increases the endogenous jasmonic acid level, PR gene expression, and host resistance to fungal infection. Mol Plant-Microbe Interact. 2006;19:1127-37.

49. Yang Y, Qi M, Mei C. Endogenous salicylic acid protects rice plants from oxidative damage caused by aging as well as biotic and abiotic stress. Plant J. 2004;40(6):909-19

50. Wasternack C, Hause B. Jasmonates: biosynthesis, perception, signal transduction and action in plant stress response, growth and development. Ann Bot. 2013;111:1021-58

51. Park C-H, Chen S, Shirsekar G, Zhou B, Khang CH, Songkumarn P, Afzal AJ, Ning $Y$, Wang R, Bellizzi M, et al. The Magnaporthe oryzae effector AvrPiz-t targets the RING E3 ubiquitin ligase APIP6 to suppress pathogen-associated molecular pattern-triggered immunity in rice. Plant Cell. 2012;24(11):4748-62.

52. Kauffman HE, Reddy APK, Hsieh SPY, Merca SD. An improved technique for evaluating resistance to rice varieties of Xanthomonas oryzae pv. Oryzae. Plant Dis Rep. 1973;57:537-41.

53. Chern M, Canlas PE, Fitzgerald HA, Ronald PC. Rice NRR, a negative regulator of disease resistance, interacts with Arabidopsis NPR1 and rice NH1. Plant J. 2005;43:623-35

54. Ou J, Peng Y, Yang W, Zhang Y, Hao J, Li F, Chen Y, Zhao Y, Xie X, Wu S, et al. ABHD5 blunts the sensitivity of colorectal cancer to fluorouracil via promoting autophagic uracil yield. Nat Commun. 2019;10(1):1078.

55. Fu J, Chu J, Sun X, Wang J, Yan C. Simple, rapid, and simultaneous assay of multiple carboxyl containing Phytohormones in wounded tomatoes by
UPLC-MS/MS using single SPE purification and isotope dilution. Anal Sci. 2012;28(11):1081-7.

56. Akhter D, Qin R, Nath UK, Alamin M, Jin X, Shi C. The Brown Midrib Leaf (bml) Mutation in Rice (Oryza sativa L.) Causes Premature Leaf Senescence and the Induction of Defense Responses. Genes. 2018;9(4):203.

57. Yang C, Yu Y, Huang J, Meng F, Pang J, Zhao Q, Islam MA, Xu N, Tian Y, Liu J. Binding of the Magnaporthe oryzae chitinase MoChia1 by a rice tetratricopeptide repeat protein allows free chitin to trigger immune responses. Plant Cell. 2019;31(1):172-88.

58. Trapnell C, Williams BA, Pertea G, Mortazavi A, Kwan G. Baren MJv, Salzberg SL, Wold BJ, Pachter L: transcript assembly and quantification by RNA-Seq reveals unannotated transcripts and isoform switching during cell differentiation. Nat Biotechnol. 2010;28(5):511-5.

\section{Publisher's Note}

Springer Nature remains neutral with regard to jurisdictional claims in published maps and institutional affiliations.
Ready to submit your research? Choose BMC and benefit from:

- fast, convenient online submission

- thorough peer review by experienced researchers in your field

- rapid publication on acceptance

- support for research data, including large and complex data types

- gold Open Access which fosters wider collaboration and increased citations

- maximum visibility for your research: over $100 \mathrm{M}$ website views per year

At BMC, research is always in progress.

Learn more biomedcentral.com/submissions 